\title{
Achieving Optimality in Robust Joint Optimization of Linear Transceiver Design
}

\author{
Hongying Tang, Wen Chen, Senior Member, IEEE, \\ Jun Li, Member, IEEE
}

\begin{abstract}
This paper presents new results on linear transceiver designs in a multiple-input-multiple-output (MIMO) link. By considering the minimal total mean-square error (MSE) criterion, we prove that the robust optimal linear transceiver design has a channel-diagonalizing structure, which verifies the conjecture in the previous work [1]. Based on this property, the original design problem can be transformed into a scalar problem, whose global optimal solution is first obtained in this work. Simulation results show the performance advantages of our solution over the existing schemes.
\end{abstract}

Index Terms-Robust design, mean-square error (MSE), linear transceiver design, convex optimization

\section{INTRODUCTION}

MIMO technique has attracted a considerable interest from both academic and industrial fields in recent years. By exploiting the multiplexing and diversity property, it can significantly improve the spectral efficiency and link reliability of the system [2]. In the literatures, transceiver designs in MIMO systems have been extensively studied in [1]-[13]. One approach of the designs is to allow nonlinear process at the transmitter or the receiver, such as the successive interference cancelation receiver design discussed by [2], or the Maximum Likelihood detector investigated in [3], [4].

As an alternative approach, the linear transceiver design, which only allows linear matrix multiplication of the signal, is more preferable in a practical system due to low implementation complexity, and is the focus of this paper. In [5], the joint optimal linear transceiver design problem was addressed, and a closed-form solution was derived. Their result was generalized into the multicarrier MIMO system in [6], by developing a unified optimization framework. The aforementioned works [5], [6] enjoy a common favorable feature that the transceiver processing matrix parallelized the original channel and allocated power to each data stream. In light of the optimality of this channel-diagonalizing structure in the perfect channel state information (CSI) case, one may

Copyright (c) 2015 IEEE. Personal use of this material is permitted. However, permission to use this material for any other purposes must be obtained from the IEEE by sending a request to pubs-permissions@ieee.org.

Hongying Tang and Wen Chen are with the Department of Electronic Engineering, Shanghai Jiaotong University, Shanghai, and School of Electronic Engineering and Automation, Guilin University of Electronic Technology, Guilin, China. (e-mail: \{lojordan, wenchen\}@sjtu.edu.cn). Jun Li is with school of Eletrical and Information Engineering, University of Sydney, Australia. Email: jun.li1@sydney.edu.au.

This work is supported by the National 973 Project \#2012CB316106, NSF China \#61328101, by STCSM Science and Technology Innovation Program \#13510711200, and by SEU National Key Lab on Mobile Communications \#2013D11. wonder whether the same property holds for the robust design in the imperfect CSI case.

Robust design, which aims to reduce the sensitivity of the imperfect CSI to the system performance, has attracted much attention [1], [7]-[9]. Generally, there are two widely used CSI uncertainty models in the literature: the stochastic model and the deterministic model. For the statistical CSI uncertainty model, where the distribution of CSI uncertainties is assumed to be known, this channel-diagonalizing structure has been well established in MIMO channels [7], [8]. However, for the deterministic CSI uncertainty model, which assumes that the instantaneous value of CSI error is norm-bounded, this problem remains unsolved, and only some restricted results were obtained in [1], [9]. The authors in [9] proposed a semirobust scheme, by optimizing only the transmit processing matrix with some fixed equalizer. Obviously, this scheme cannot fully exploit the performance gain by the equalizer, since the fixed equalizer may not be optimal. Later in [1], the authors considered joint linear transceiver design, and showed a superior performance over [9]. By imposing certain structural constraints on the processing matrix at the transmitter or receiver side, they observed the favorable channel-diagonalizing structure. Then they transformed the original problem into the issues of power loading among each data stream, which were further solved by the alternation optimization method. However, two problems in [1] were left unsolved:

Q1) Joint Optimal structure: Without any additional structural restriction, is this channel-diagonalizing structure joint optimal?

Q2) Global Optimal solution: If it is, does the alternatingoptimization based method converge to the global optimal solution?

In this paper, we will answer the above two questions raised by [1]. Without assuming any specific structure for the linear transmitter-equalizer matrix, we show that the optimal design actually admits a channel-diagonalizing structure. Based on this property, the original problem reduces to a scalar convex problem, whose optimal solution can thus be efficiently obtained. Simulation results in section $\mathrm{V}$ show the superior performance of our solution over that in [1].

Notations: [.] ${ }^{H}$ denotes conjugate transpose of a matrix or a vector. $\mathbf{I}$ and $\mathbf{0}$ denote the identity and zero matrix, respectively. $\mathbb{R}^{N}$ and $\mathbb{C}^{N}$ respectively denote the $N$ dimensional real field and complex field. $\|\cdot\|_{2}$ and $\|\cdot\|_{F}$ denote the the Frobenius norm of a vector and a matrix, respectively. We will use boldface lowercase letters to denote column vectors and boldface uppercase letters to denote matrices. The positive 
semidefinite matrix $\mathbf{X}$ is denoted by $\mathbf{X} \succeq 0 . \operatorname{diag}\left\{\mathbf{x}_{1}, \cdots, \mathbf{x}_{R}\right\}$ denotes diagonal concatenation of block matrices $\mathbf{x}_{1}, \cdots, \mathbf{x}_{R}$. The $\operatorname{tr}(\cdot)$ is the trace of a matrix. $\operatorname{vec}(\mathbf{X})$ stacks the columns of matrix $\mathbf{X}$ into a vector. $\otimes$ denotes the Kronecker product. $\mathfrak{R}\{\cdot\}$ denotes the real part of a complex number. $\lambda_{\max }(\cdot)$ is the maximum eigenvalue of a matrix.

\section{Problem Statement}

We consider a MIMO communication system equipped with $N$ transmit antennas at the source and $M$ receive antennas at the destination. The symbol vector $\mathbf{s} \in \mathbb{C}^{L}$ is linearly precoded by a source precoding matrix $\mathbf{F} \in \mathbb{C}^{N \times L}$, through the MIMO channel $\mathbf{H} \in \mathbb{C}^{M \times N}$, and then received by the destination. We assume that $E\left\{\mathbf{s s}^{H}\right\}=\mathbf{I}$ without loss of generality. Generally, the transmitter imposes a power constraint on the precoding matrix $\mathbf{F}$ as $\operatorname{tr}\left(\mathbf{F F}{ }^{H}\right) \leq P$. A linear equalizer $\mathbf{G} \in \mathbb{C}^{L \times M}$ is usually applied on the received signal to obtain the estimated symbol vector $\hat{\mathbf{s}}$ as

$$
\hat{\mathbf{s}}=\mathbf{G H F s}+\mathbf{G n},
$$

where $\mathbf{n} \in \mathbb{C}^{M}$ is the additive white Gaussian noise (AWGN) observed at the destination with variance $\sigma_{n}^{2} \mathbf{I}$. Then the MSE between $\hat{\mathbf{s}}$ and $\mathbf{s}$ is given by

$$
\mathrm{MSE} \triangleq \mathcal{E}\left\{\|\hat{\mathbf{s}}-\mathbf{s}\|^{2}\right\}=\|\mathbf{G H F}-\mathbf{I}\|_{F}^{2}+\sigma_{n}^{2}\|\mathbf{G}\|_{F}^{2} .
$$

We assume that $L \leq \operatorname{rank}(\mathbf{H})$, since the number of degrees of freedom is upper bounded by $L \leq \operatorname{rank}(\mathbf{H})=\min \{M, N\}$.

In a practical wireless communication scenario, perfect CSI is usually difficult to obtain. With only imperfect CSI, the system performance will be deteriorated. This motivates us to investigate the robust design taking the CSI errors into account. To characterize the mismatched CSI, we adopt a common deterministic imperfect CSI model [1], [9], and write the channel matrix as

$$
\mathbf{H}=\tilde{\mathbf{H}}+\mathbf{E},
$$

where $\tilde{\mathbf{H}}$ is the estimated channel matrix and $\mathbf{E}$ is the corresponding CSI error matrix satisfying $\|\mathbf{E}\|_{F} \leq \varepsilon$ for some $\varepsilon \geq 0$. As in [1], [9], we assume that only $\tilde{\mathbf{H}}$ and $\varepsilon$ are available at both ends.

By taking into imperfect CSI model (1) into account, the robust transmitter-equalizer design is given by the solution of the following min-max problem:

$$
\begin{aligned}
\min _{\mathbf{G}, \mathbf{F}} \max _{\|\mathbf{E}\|_{F} \leq \varepsilon} & \|\mathbf{G}(\tilde{\mathbf{H}}+\mathbf{E}) \mathbf{F}-\mathbf{I}\|_{F}^{2}+\sigma_{n}^{2}\|\mathbf{G}\|_{F}^{2}, \\
\text { s.t. } & \operatorname{tr}\left(\mathbf{F F} \mathbf{F}^{H}\right) \leq P .
\end{aligned}
$$

\section{Robust Joint Optimal Structure of F And $\mathbf{G}$}

In this section, we will determine the joint optimal structure of $\mathbf{F}$ and $\mathbf{G}$ in problem (2), showing that they diagonalize the MIMO channel into eigen subchannels. We also figure out that the worst-case CSI uncertainty $\mathbf{E}$ has the similar singular value decomposition (SVD) structure as the nominal channel $\tilde{\mathbf{H}}$, which simplifies problem (2) into a scalar problem as we will shown in section IV.

Denote the SVD structure of $\mathbf{F}$ and $\mathbf{G}$ by $\mathbf{F}=\mathbf{U}_{f} \boldsymbol{\Sigma}_{f} \mathbf{V}_{f}^{H}$ and $\mathbf{G}=\mathbf{U}_{g} \boldsymbol{\Sigma}_{g} \mathbf{V}_{g}^{H}$, respectively, where $\mathbf{U}_{f}, \mathbf{V}_{f}, \mathbf{U}_{g}$ and $\mathbf{V}_{g}$ are unitary matrices. The matrices $\boldsymbol{\Sigma}_{f}$ and $\boldsymbol{\Sigma}_{g}$ can be written as

$$
\boldsymbol{\Sigma}_{f}=\left[\hat{\boldsymbol{\Sigma}}_{f}, \mathbf{0}\right]^{T}, \boldsymbol{\Sigma}_{g}=\left[\hat{\boldsymbol{\Sigma}}_{g}, \mathbf{0}\right]
$$

where $\hat{\boldsymbol{\Sigma}}_{f} \triangleq \operatorname{diag}\left\{f_{1}, \cdots, f_{L}\right\}$ and $\hat{\boldsymbol{\Sigma}}_{g} \triangleq \operatorname{diag}\left\{g_{1}, \cdots, g_{L}\right\}$ are real diagonal matrices. Denote the nominal channel $\tilde{\mathbf{H}}$ by $\tilde{\mathbf{H}}=\mathbf{U}_{h} \boldsymbol{\Sigma}_{h} \mathbf{V}_{h}^{H}$ and let $\hat{\boldsymbol{\Sigma}}_{h}$ be the $L \times L$ diagonal matrix containing the largest $L$ singular values $\gamma_{1} \geq \cdots \geq \gamma_{L}$. Then the following theorem determines the optimal structure of $\mathbf{F}$ and $\mathbf{G}$.

Theorem 1: The robust optimal $\mathrm{F}$ and $\mathrm{G}$ in problem (2) can be expressed in the following structure:

$$
\begin{aligned}
\mathbf{F} & =\mathbf{V}_{h} \boldsymbol{\Sigma}_{f}, \\
\mathbf{G} & =\boldsymbol{\Sigma}_{g} \mathbf{U}_{h}^{H} .
\end{aligned}
$$

Meanwhile the corresponding worst case channel uncertainty is given by $\mathbf{E}=\mathbf{U}_{h} \boldsymbol{\Delta}_{D} \mathbf{V}_{h}^{H}$, with $\boldsymbol{\Delta}_{D}=\operatorname{diag}\left\{\hat{\boldsymbol{\Delta}}_{D}, \mathbf{0}\right\}$, and $\hat{\boldsymbol{\Delta}}_{D} \in \mathbb{R}^{L \times L}$ being diagonal.

Proof: We write the first additive term of the objective function of (2) as

$$
\begin{aligned}
\|\mathbf{G}(\hat{\mathbf{H}}+\mathbf{E}) \mathbf{F}-\mathbf{I}\|_{F}^{2} & \stackrel{(a)}{=}\left\|\mathbf{G} \mathbf{U}_{h}\left(\boldsymbol{\Sigma}_{h}+\boldsymbol{\Delta}\right) \mathbf{V}_{h}^{H} \mathbf{F}-\mathbf{I}\right\|_{F}^{2} \\
& \stackrel{(b)}{=}\left\|\mathbf{G}^{\prime}\left(\boldsymbol{\Sigma}_{h}+\boldsymbol{\Delta}\right) \mathbf{F}^{\prime}-\mathbf{I}\right\|_{F}^{2},
\end{aligned}
$$

where in $(a)$ we have defined $\boldsymbol{\Delta} \triangleq \mathbf{U}_{h}^{H} \mathbf{E V}_{h}$. By the unitaryinvariant property of $\|\cdot\|_{F}$, we can see that $\Delta$ still satisfies $\|\boldsymbol{\Delta}\|_{F} \leq \varepsilon$. In $(b)$ we have defined $\mathbf{G}^{\prime} \triangleq \mathbf{G U}_{h}$ and $\mathbf{F}^{\prime} \triangleq$ $\mathbf{V}_{h}^{H} \mathbf{F}$. Now problem (2) can be rewritten as

$$
\begin{aligned}
\min _{\mathbf{G}^{\prime}, \mathbf{F}^{\prime}} \max _{\|\boldsymbol{\Delta}\|_{F} \leq \varepsilon} & \operatorname{MSE}\left(\mathbf{F}^{\prime}, \mathbf{G}^{\prime}, \boldsymbol{\Delta}\right) \\
& \triangleq\left\|\mathbf{G}^{\prime}\left(\boldsymbol{\Sigma}_{h}+\boldsymbol{\Delta}\right) \mathbf{F}^{\prime}-\mathbf{I}\right\|_{F}^{2}+\sigma_{d}^{2}\left\|\mathbf{G}^{\prime}\right\|_{F}^{2}, \\
\text { s.t. } & \operatorname{tr}\left(\mathbf{F}^{\prime} \mathbf{F}^{\prime H}\right) \leq P
\end{aligned}
$$

which is an optimization problem with respect to $\mathbf{F}^{\prime}$ and $\mathbf{G}^{\prime}$. To proceed, we first discuss a particular case when $\left(\mathbf{F}^{\prime}, \mathbf{G}^{\prime}\right)=$ $\left(\left[\hat{\boldsymbol{\Sigma}}_{f}, \mathbf{0}\right]^{T},\left[\hat{\boldsymbol{\Sigma}}_{g}, \mathbf{0}\right]\right)$. Then problem (5) becomes

$$
\begin{aligned}
\min _{\hat{\boldsymbol{\Sigma}}_{g}, \hat{\boldsymbol{\Sigma}}_{f}\|\hat{\boldsymbol{\Delta}}\|_{F} \leq \varepsilon} & \left\|\hat{\boldsymbol{\Sigma}}_{g}\left(\hat{\boldsymbol{\Sigma}}_{h}+\hat{\boldsymbol{\Delta}}\right) \hat{\boldsymbol{\Sigma}}_{f}-\mathbf{I}\right\|_{F}^{2}+\sigma_{d}^{2}\left\|\hat{\boldsymbol{\Sigma}}_{g}\right\|_{F}^{2}, \\
\text { s.t. } & \operatorname{tr}\left(\hat{\boldsymbol{\Sigma}}_{f} \hat{\boldsymbol{\Sigma}}_{f}^{H}\right) \leq P
\end{aligned}
$$

where $\hat{\Delta}$ is the upper left $L \times L$ submatrix of $\Delta$. We will then show that there exist an optimal $\hat{\Delta}$ in (6) that is diagonal.

After some matrix manipulations and noticing the fact that the maximization of a convex function is achieved on the boundary [9], the inner maximization of problem (6) can be transformed into the following problem

$$
\min _{\|\boldsymbol{\delta}\|=\varepsilon} \boldsymbol{\delta}^{H}\left(-\mathbf{B}^{T} \otimes \mathbf{C}\right) \boldsymbol{\delta}-2 \mathfrak{R}\left\{\mathbf{d}^{H} \boldsymbol{\delta}\right\},
$$

where $\boldsymbol{\delta} \triangleq \operatorname{vec}(\hat{\boldsymbol{\Delta}}), \mathbf{C} \triangleq \hat{\boldsymbol{\Sigma}}_{g} \hat{\boldsymbol{\Sigma}}_{g}^{H}, \mathbf{B} \triangleq \hat{\boldsymbol{\Sigma}}_{f} \hat{\boldsymbol{\Sigma}}_{f}^{H}$, and $\mathbf{d} \triangleq$ $\operatorname{vec}\left(\hat{\boldsymbol{\Sigma}}_{g}^{H}\left(\hat{\boldsymbol{\Sigma}}_{g} \hat{\boldsymbol{\Sigma}}_{h} \hat{\boldsymbol{\Sigma}}_{f}-\mathbf{I}\right) \hat{\boldsymbol{\Sigma}}_{f}^{H}\right)$.

By the result in [9], $\delta$ is a global minimizer of (7) if and only if there exists an $\omega$ such that

$$
\left(-\mathbf{B}^{T} \otimes \mathbf{C}+\omega \mathbf{I}\right) \boldsymbol{\delta}=\mathbf{d},-\mathbf{B}^{T} \otimes \mathbf{C}+\omega \mathbf{I} \succeq \mathbf{0},\|\boldsymbol{\delta}\|=\varepsilon,
$$


which is equivalent to

$$
\begin{aligned}
\omega \hat{\boldsymbol{\Delta}}-\mathbf{C} \hat{\boldsymbol{\Delta}} \mathbf{B} & =\hat{\boldsymbol{\Sigma}}_{g}^{H}\left(\hat{\boldsymbol{\Sigma}}_{g} \hat{\boldsymbol{\Sigma}}_{h} \hat{\boldsymbol{\Sigma}}_{f}-\mathbf{I}\right) \hat{\boldsymbol{\Sigma}}_{f}^{H} \\
\operatorname{tr}\left(\hat{\boldsymbol{\Delta}} \hat{\boldsymbol{\Delta}}^{H}\right) & =\varepsilon^{2} \\
\omega & \geq \lambda_{\max }\left(\mathbf{B}^{T} \otimes \mathbf{C}\right) .
\end{aligned}
$$

Since both $\mathbf{C}$ and $\mathbf{B}$ are diagonal, (8)-(10) tells us that for any given $\hat{\boldsymbol{\Sigma}}_{f}$ and $\hat{\boldsymbol{\Sigma}}_{g}$, there exists an optimal $\hat{\boldsymbol{\Delta}}$ that is diagonal. Denote the optimal solution of (6) as $\left(\hat{\boldsymbol{\Sigma}}_{f}^{\sharp}, \hat{\boldsymbol{\Sigma}}_{g}^{\sharp}, \hat{\boldsymbol{\Delta}}_{D}^{\sharp}\right)$ with $\hat{\boldsymbol{\Delta}}_{D}^{\sharp}$ being diagonal. To facilitate the analysis, we further define $\mathbf{F}^{\prime \sharp} \triangleq\left[\hat{\boldsymbol{\Sigma}}_{f}^{\sharp}, \mathbf{0}\right]^{T}, \mathbf{G}^{\prime \sharp} \triangleq\left[\hat{\boldsymbol{\Sigma}}_{g}^{\sharp}, \mathbf{0}\right]$ and $\boldsymbol{\Delta}_{D}^{\sharp} \triangleq \operatorname{diag}\left\{\hat{\boldsymbol{\Delta}}_{D}^{\sharp}, \mathbf{0}\right\}$. Then by the above definitions and discussions, we have

$$
\max _{\hat{\mathbf{\Delta}}{ }_{F} \leq \varepsilon} \operatorname{MSE}\left(\mathbf{F}^{\prime \sharp}, \mathbf{G}^{\prime \sharp}, \boldsymbol{\Delta}\right)=\operatorname{MSE}\left(\mathbf{F}^{\prime \sharp}, \mathbf{G}^{\prime \sharp}, \boldsymbol{\Delta}_{D}^{\sharp}\right) .
$$

Now we discuss another particular situation when $\Delta=$ $\boldsymbol{\Delta}_{D}^{\sharp}$. Then problem (5) becomes

$$
\begin{aligned}
\min _{\mathbf{F}^{\prime}, \mathbf{G}^{\prime}} & \left\|\mathbf{G}^{\prime}\left(\boldsymbol{\Sigma}_{h}+\boldsymbol{\Delta}_{D}^{\sharp}\right) \mathbf{F}^{\prime}-\mathbf{I}\right\|_{F}^{2}+\sigma_{d}^{2}\left\|\mathbf{G}^{\prime}\right\|_{F}^{2}, \\
\text { s.t. } & \operatorname{tr}\left(\mathbf{F}^{\prime} \mathbf{F}^{\prime H}\right) \leq P .
\end{aligned}
$$

which has been discussed in [5], [7], [10], and the optimal solution is given by

$$
\left(\mathbf{F}^{\prime}, \mathbf{G}^{\prime}\right)=\left(\left[\hat{\boldsymbol{\Sigma}}_{f}, \mathbf{0}\right]^{T},\left[\hat{\boldsymbol{\Sigma}}_{g}, \mathbf{0}\right]\right)
$$

Substituting (13) into problem (12), we have

$$
\begin{aligned}
\min _{\hat{\boldsymbol{\Sigma}}_{f}, \hat{\boldsymbol{\Sigma}}_{g}} & \left\|\hat{\boldsymbol{\Sigma}}_{g}\left(\hat{\boldsymbol{\Sigma}}_{h}+\hat{\boldsymbol{\Delta}}_{D}^{\sharp}\right) \hat{\boldsymbol{\Sigma}}_{f}-\mathbf{I}\right\|_{F}^{2}+\sigma_{d}^{2}\left\|\hat{\boldsymbol{\Sigma}}_{g}\right\|_{F}^{2}, \\
\text { s.t. } & \operatorname{tr}\left(\hat{\boldsymbol{\Sigma}}_{f} \hat{\boldsymbol{\Sigma}}_{f}^{H}\right) \leq P .
\end{aligned}
$$

Remember that the optimal solution of (6) is denoted by $\left(\hat{\boldsymbol{\Sigma}}_{f}^{\sharp}, \hat{\boldsymbol{\Sigma}}_{g}^{\sharp}, \hat{\boldsymbol{\Delta}}_{D}^{\sharp}\right)$. Then it is easy to know that the optimal solution of (14) is given by $\left(\hat{\Sigma}_{f}^{\sharp}, \hat{\Sigma}_{g}^{\sharp}\right)$, which means that when the channel uncertainty is $\boldsymbol{\Delta}_{D}^{\sharp}=\operatorname{diag}\left\{\hat{\boldsymbol{\Delta}}_{D}^{\sharp}, \mathbf{0}\right\}$, the optimal $\left(\mathbf{F}^{\prime}, \mathbf{G}^{\prime}\right)$ of problem (12) is given by $\left(\left[\hat{\boldsymbol{\Sigma}}_{f}^{\sharp}, \mathbf{0}\right]^{T},\left[\hat{\boldsymbol{\Sigma}}_{g}^{\sharp}, \mathbf{0}\right]\right)$, or we have

$$
\operatorname{MSE}\left(\mathbf{F}^{\prime}, \mathbf{G}^{\prime}, \boldsymbol{\Delta}_{D}^{\sharp}\right) \geq \operatorname{MSE}\left(\mathbf{F}^{\prime \sharp}, \mathbf{G}^{\prime \sharp}, \boldsymbol{\Delta}_{D}^{\sharp}\right) .
$$

We will next show that from these two special cases given in (11) and (15), the joint optimal structure of $\mathbf{F}^{\prime}$ and $\mathbf{G}^{\prime}$ can be obtained. This technique has also been used in [11]-[13], and is detailed as follows

$$
\begin{aligned}
& \max _{\|\boldsymbol{\Delta}\|_{F} \leq \varepsilon} \operatorname{MSE}\left(\mathbf{F}^{\prime}, \mathbf{G}^{\prime}, \boldsymbol{\Delta}\right) \stackrel{(a)}{\geq} \operatorname{MSE}\left(\mathbf{F}^{\prime}, \mathbf{G}^{\prime}, \boldsymbol{\Delta}_{D}^{\sharp}\right) \\
& \stackrel{(b)}{\geq} \operatorname{MSE}\left(\mathbf{F}^{\prime \sharp}, \mathbf{G}^{\prime \sharp}, \boldsymbol{\Delta}_{D}^{\sharp}\right) \stackrel{(c)}{=} \max _{\|\boldsymbol{\Delta}\|_{F} \leq \varepsilon} \operatorname{MSE}\left(\mathbf{F}^{\prime \sharp}, \mathbf{G}^{\prime \sharp}, \boldsymbol{\Delta}\right)
\end{aligned}
$$

where $(a)$ is due to the fact that $\Delta_{D}^{\sharp}$ is only a particular channel, $(b)$ is due to (15) and $(c)$ is due to (11). Inequality (16) shows that the optimal $\mathbf{F}^{\prime}$ and $\mathbf{G}^{\prime}$ must be given by $\left(\mathbf{F}^{\prime \sharp}, \mathbf{G}^{\prime \sharp}\right)$. The proof is completed.

Remark 1: Theorem 1 provides some interesting insights into the robust optimal transceiver design, showing that its optimal structure is given by $\mathbf{V}_{f}=\mathbf{U}_{g}, \mathbf{U}_{f}=\mathbf{V}_{h}$ and $\mathbf{V}_{g}=\mathbf{U}_{h}$, which diagonalizes the MIMO channel into eigen subchannels, and is consistent with the results under perfect and stochastic CSI assumptions. Therefore, the answer to question $\mathcal{Q} 1$ is yes! Note that problem (2) was also considered in [1], where only partial results of Theorem 1 was obtained. That is, by assuming $\mathbf{V}_{g}=\mathbf{U}_{h}$, then $\mathbf{V}_{f}=\mathbf{U}_{g}$ and $\mathbf{U}_{f}=\mathbf{V}_{h}$ were proved to be optimal; on the other hand, given $\mathbf{U}_{f}=\mathbf{V}_{h}$, then $\mathbf{V}_{f}=\mathbf{U}_{g}$ and $\mathbf{V}_{g}=\mathbf{U}_{h}$ were proved to be optimal.

\section{Robust Global Optimal Design Based ON SCALAR OPTIMIZATION}

Based on Theorem 1, we know that the optimal solution of problem (2) is determined by problem (6), where $\hat{\Delta}$ is diagonal. Denote $\hat{\boldsymbol{\Delta}} \triangleq \operatorname{diag}\left\{x_{1}, \cdots, x_{L}\right\}, \mathbf{f} \triangleq\left[f_{1}, \cdots, f_{L}\right]^{T}$ and $\mathbf{g} \triangleq\left[g_{1}, \cdots, g_{L}\right]^{T}$, then problem (6) is equivalent to

$$
\begin{aligned}
\min _{\mathbf{f}, \mathbf{g} \sum_{i=1}^{L} x_{i}^{2} \leq \varepsilon^{2}} & \max _{i=1}^{L}\left(f_{i} g_{i}\left(\gamma_{i}+x_{i}\right)-1\right)^{2}+\sigma_{n}^{2} \sum_{i=1}^{L} g_{i}^{2} \\
\text { s.t. } & \sum_{i=1}^{L} f_{i}^{2} \leq P .
\end{aligned}
$$

Introducing a slack variable $t$, problem 17 can be converted to

$$
\begin{array}{ll}
\min _{\mathbf{f}, \mathbf{g}, t} & t+\sigma_{n}^{2} \sum_{i=1}^{L} g_{i}^{2} \\
\text { s.t. } & \sum_{i=1}^{L}\left(f_{i} g_{i}\left(\gamma_{i}+x_{i}\right)-1\right)^{2} \leq t, \sum_{i=1}^{L} x_{i}^{2} \leq \varepsilon^{2} \\
& \sum_{i=1}^{L} f_{i}^{2} \leq P .
\end{array}
$$

Generally speaking, it is difficult to derive a closedform solution of (18). Thus we will solve it in numerical results. Let $\boldsymbol{\eta} \triangleq\left[f_{1} g_{1} \gamma_{1}-1, \cdots, f_{L} g_{L} \gamma_{L}-1\right]^{T}, \boldsymbol{\Gamma} \triangleq$ $\operatorname{diag}\left\{f_{1} g_{1}, \cdots, f_{L} g_{L}\right\}$, and $\mathbf{x} \triangleq\left[x_{1}, \cdots, x_{L}\right]^{T}$. Constraint (18b) can be rewritten as

$$
\|\boldsymbol{\eta}+\boldsymbol{\Gamma} \mathbf{x}\|_{2}^{2} \leq t,\|\mathbf{x}\|_{2} \leq \varepsilon .
$$

Following the similar lines in [1], [9], one can transform (19] into

$$
\left[\begin{array}{ccc}
t-\mu & \boldsymbol{\eta}^{H} & \mathbf{0} \\
\boldsymbol{\eta} & \mathbf{I} & \varepsilon \boldsymbol{\Gamma} \\
\mathbf{0} & \varepsilon \boldsymbol{\Gamma}^{H} & \mu \mathbf{I}
\end{array}\right] \succeq \mathbf{0}, \exists \mu \geq 0 .
$$

By applying Schur's Complement [14], (20) is equivalent to the following constraint

$$
\begin{aligned}
{\left[\begin{array}{cc}
t-\mu & \boldsymbol{\eta}^{H} \\
\boldsymbol{\eta} & \mathbf{I}
\end{array}\right] } & -\frac{1}{\mu}\left[\begin{array}{c}
\mathbf{0} \\
\varepsilon \boldsymbol{\Gamma}
\end{array}\right]\left[\begin{array}{lc}
\mathbf{0} & \varepsilon \boldsymbol{\Gamma}^{H}
\end{array}\right] \\
& =\left[\begin{array}{cc}
t-\mu & \boldsymbol{\eta}^{H} \\
\boldsymbol{\eta} & \mathbf{I}-\frac{1}{\mu} \varepsilon^{2} \boldsymbol{\Gamma} \boldsymbol{\Gamma}^{H}
\end{array}\right] \succeq \mathbf{0} .
\end{aligned}
$$

Using Schur's Complement again, (21) can be written as

$$
t-\mu-\boldsymbol{\eta}^{H}\left(\mathbf{I}-\frac{1}{\mu} \varepsilon^{2} \boldsymbol{\Gamma} \boldsymbol{\Gamma}^{H}\right)^{-1} \boldsymbol{\eta} \geq \mathbf{0}
$$

or equivalently

$$
\mu+\sum_{i=1}^{L} \frac{\left(f_{i} g_{i} \gamma_{i}-1\right)^{2}}{1-\varepsilon^{2} f_{i}^{2} g_{i}^{2} / \mu} \leq t .
$$


Combining (18) and (22), we get the following problem

$$
\begin{aligned}
\min _{\mathbf{f}, \mathbf{g}, \mu} & \sum_{i=1}^{L} \frac{\left(\gamma_{i} f_{i} g_{i}-1\right)^{2}}{1-\varepsilon^{2} f_{i}^{2} g_{i}^{2} / \mu}+\mu+\sigma_{n}^{2} \sum_{i=1}^{L} g_{i}^{2} \\
\text { s.t. } & \sum_{i=1}^{L} f_{i}^{2} \leq P, \mu \geq \varepsilon^{2} f_{i}^{2} g_{i}^{2},
\end{aligned}
$$

where the constraint $\mu \geq \varepsilon^{2} f_{i}^{2} g_{i}^{2}$ is implicitly included in the constraint (21).

Problem (23) is still difficult to deal with. However, we will next show that by some variable transformations, the global optimal solution of problem (23) can be obtained. Define $\mathbf{m} \triangleq$ $\left[m_{1}, \cdots, m_{L}\right]^{T}$ and $\mathbf{n} \triangleq\left[n_{1}, \cdots, n_{L}\right]^{T}$, where $m_{i}=f_{i} g_{i}$ and $n_{i}=g_{i}^{2}$, for $i=1, \cdots, L$. Then problem (23) becomes

$$
\begin{aligned}
\min _{\mathbf{m}, \mathbf{n}, \mu} & \phi_{1}(\mathbf{m}, \mathbf{n}, \mu) \triangleq \sum_{i=1}^{L} \frac{\left(\gamma_{i} m_{i}-1\right)^{2}}{1-\varepsilon^{2} m_{i}^{2} / \mu}+\mu+\sigma_{n}^{2} \sum_{i=1}^{L} n_{i} \\
\text { s.t. } & \sum_{i=1}^{L} m_{i}^{2} / n_{i} \leq P, \mu \geq \varepsilon^{2} m_{i}^{2},
\end{aligned}
$$

Let $\mathbf{s} \triangleq\left[s_{1}, \cdots, s_{L}\right]^{T}$. We claim that (24) is equivalent to the following problem

$$
\begin{aligned}
\min _{\mathbf{m}, \mathbf{n}, \mu, \mathbf{s}} & \phi_{2}(\mathbf{m}, \mathbf{n}, \mu, \mathbf{s}) \triangleq \sum_{i=1}^{L} \frac{\left(\gamma_{i} m_{i}-1\right)^{2}}{1-s_{i}}+\mu+\sigma_{n}^{2} \sum_{i=1}^{L} n_{i} \\
\text { s.t. } & \sum_{i=1}^{L} m_{i}^{2} / n_{i} \leq P, \varepsilon^{2} m_{i}^{2} / \mu \leq s_{i}<1 .
\end{aligned}
$$

This can be explained as follows. First, suppose that $\left(\mathbf{m}^{\sharp}, \mathbf{n}^{\sharp}, \mu^{\sharp}, \mathbf{s}^{\sharp}\right)$ is the optimal solution of (25). In view of (24), it follows that

$$
\begin{aligned}
\min _{\mathbf{m}, \mathbf{n}, \mu} \phi_{1}(\mathbf{m}, \mathbf{n}, \mu) \leq \phi_{1}\left(\mathbf{m}^{\sharp}, \mathbf{n}^{\sharp}, \mu^{\sharp}\right) \\
\stackrel{(a)}{\leq} \phi_{2}\left(\mathbf{m}^{\sharp}, \mathbf{n}^{\sharp}, \mu^{\sharp}, \mathbf{s}^{\sharp}\right)=\min \phi_{2}(\mathbf{m}, \mathbf{n}, \mu, \mathbf{s}),
\end{aligned}
$$

where in $(a)$ we have used the constraint $\varepsilon^{2} m_{i}^{\sharp 2} / \mu^{\sharp} \leq s_{i}^{\sharp}$. Then we know that $\min \phi_{1}(\mathbf{m}, \mathbf{n}, \mu) \leq \min \phi_{2}(\mathbf{m}, \mathbf{n}, \mu, \mathbf{s})$. On the other hand, for any feasible $(\mathbf{m}, \mathbf{n}, \mu)$ of (24), we can always find some $\mathbf{s}$, which makes the equality holds in (25). This means that, it is also feasible to 25]. Then we must have $\min \phi_{1}(\mathbf{m}, \mathbf{n}, \mu) \geq \min \phi_{2}(\mathbf{m}, \mathbf{n}, \mu, \mathbf{s})$. Thereby we must have $\min \phi_{1}(\mathbf{m}, \mathbf{n}, \mu)=\min \phi_{2}(\mathbf{m}, \mathbf{n}, \mu, \mathbf{s})$.

Introducing the slack variable $\mathbf{z} \triangleq\left[z_{1}, \cdots, z_{L}\right]^{T}$, problem (25) can be written as

$$
\begin{aligned}
\min _{\mathbf{m}, \mathbf{n}, \mu, \mathbf{s}, \mathbf{z}} & \sum_{i=1}^{L} z_{i}+\mu+\sigma_{n}^{2} \sum_{i=1}^{L} n_{i} \\
\text { s.t. } \quad & {\left[\begin{array}{cc}
z_{i} & \gamma_{i} m_{i}-1 \\
\gamma_{i} m_{i}-1 & 1-s_{i}
\end{array}\right] \succeq \mathbf{0}, i=1, \cdots, L } \\
& {\left[\begin{array}{cc}
\mu & \varepsilon m_{i} \\
\varepsilon m_{i} & s_{i}
\end{array}\right] \succeq \mathbf{0}, s_{i}<1, i=1, \cdots, L } \\
& \sum_{i=1}^{L} \frac{m_{i}^{2}}{n_{i}} \leq P .
\end{aligned}
$$

Problem (26) is a semidefinite programming (SDP) problem, which can be efficiently solved by the MATLAB package tools such as CVX [15]. Then the optimal $f_{i}$ and $g_{i}$ are obtained by $f_{i}=m_{i} / \sqrt{n_{i}}$ and $g_{i}=\sqrt{n_{i}}$.

Remark 2: By fixing $\mathbf{f}$ or $\mathbf{g}$, problem (23) becomes problem (6) or (7) in [1], where they were proved to be convex and can be optimally solved, respectively. This process is repeated until convergence. However, the solution of this alternating optimization based method depends on the initial point $\mathbf{f}^{(0)}$, and may not be optimal if problem (23) has local minimal. Thus it is natural to ask question $\mathcal{Q} 2$, does this method converge to the global optimal solution? Unfortunately, this is not guaranteed. As will be seen in section $\mathrm{V}$ different initial point $\mathbf{f}^{(0)}$ will lead to different results, and may also incur some performance loss. Therefore, our answer to question $\mathcal{Q} 2$ is not always, and it depends on the initial point.

\section{Discussions And Simulations}

In this section, we first provide the complexity comparison between our joint optimal design and the robust design in [1], and then give numerical results to compare the two robust designs.

The channel fading is modeled as Rayleigh fading, and each channel entry satisfies the complex normal distribution $\mathcal{C N}(0,1)$. The noise is assumed to be zero-mean unit variance complex Gaussian random variables. In our simulations, we set $M=N=L$ and vary $\varepsilon$ through the normalized parameter $\rho \in[0,1)$, i.e., $\varepsilon^{2}=\rho\|\tilde{\mathbf{H}}\|_{F}^{2}$. Then the larger the $\rho$ is, the poorer the CSI quality will be. All results are averaged over 1000 channel realizations.

\section{A. Complexity Comparison}

Since we have derived the optimal structure of tranceiver design in Theorem 1, the major computing step in our work remains in solving problem (26). The complexity for solving the SDP problem (26) is $\mathcal{O}\left(L^{2}\right)$ per iteration and the number of iterations typically lies between 5 and 50, for an SDP problem [16]. On the other hand, the complexity analysis of the method in [1] is a little complicated. In their work, when fixing $\mathbf{g}$, the optimal $\mathbf{f}$ was obtained by the three-level primalprimal decomposition method, where a close-form solution was given in the lowest level, while the bisection method and the gradient method were applied at the middle and third level, respectively. Similarly, the problem for determining optimal $\mathrm{g}$ under fixed $\mathbf{f}$ was also solved in two levels, where a closeform solution was derived at the first level, while the bisection method was used at the second level. It can be seen that it is hard to determine the complexity of each iteration as well as the exact (or even approximate) iteration number in this method. Upon this observation, we resort to the CPU time comparison required by the two methods.

Fig. 1 shows the average CPU time comparison between our robust optimal method and the robust method in [1]. We set $P=20 \mathrm{dBW}$ and choose different initial points for the method in [1]: a) Scheme I: set the initial point $\mathbf{f}^{(0)}$ with equal elements. b) Scheme II: set $\mathbf{f}^{(0)}$ as the non-robust solution that takes the nominal channel $\tilde{\mathbf{H}}$ as the actual channel [5]. c) Scheme III: set $\mathbf{f}^{(0)}$ as a random variable that satisfies $\left\|\mathbf{f}^{(0)}\right\|_{2}^{2}=P$. It can be observed from Fig. 1 that scheme III 


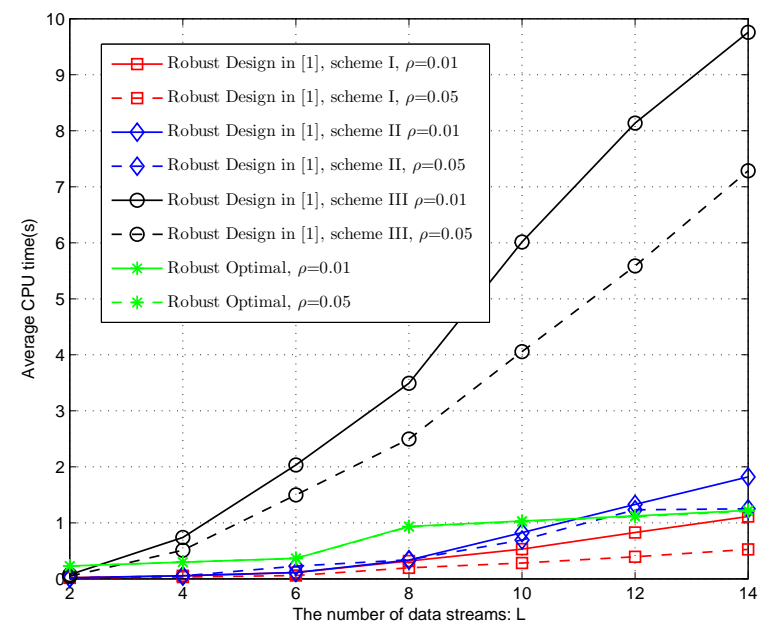

Fig. 1. Average CPU time comparison versus different $L$ for $M=N=L$ and $P=20 \mathrm{dBW}$.

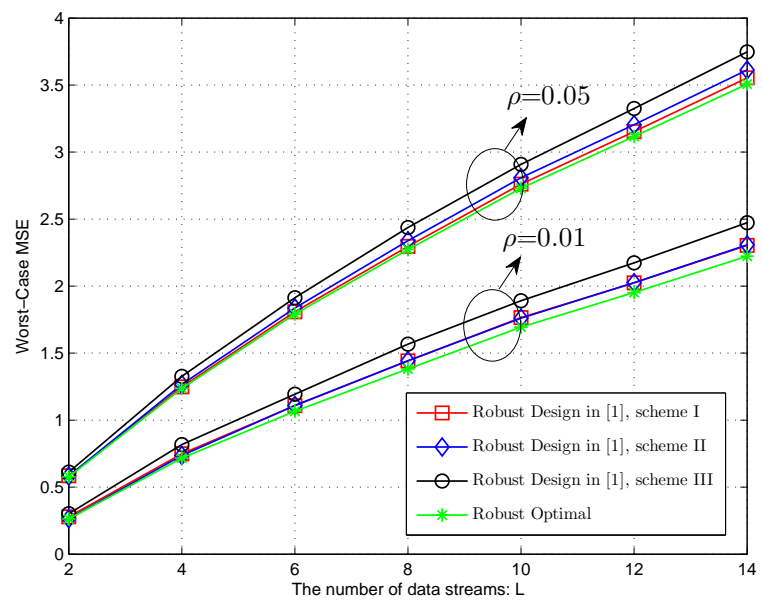

Fig. 2. Worst-case MSE versus different $L$ for $M=N=L$ and $P=$ 20dBW.

is the most time-consuming scheme among all the schemes. Although the time cost by Scheme I and II is similar to that in our our global optimal solution, it increases more rapidly as the number of data streams L goes large. From Fig. 11, we know that the required CPU time for the method in [1] tends to be more random in nature, and heavily depends on the initial point. On the other hand, our method is not only efficient but also has a more stable runtime performance.

\section{B. Numerical Results}

We now study the system MSE performance in different scenarios. In Fig. 2] we set the same network configuration as that in Fig. 1, and investigate the average worst-case MSE performance versus $L$ of our method and three different schemes in [1]. As shown in the plot, scheme I and scheme II suffer some marginal performance loss, while scheme III incurs some apparent loss, which grows even larger when $L$ increases. Hence, the method in [1] does not always lead to the optimal solution.

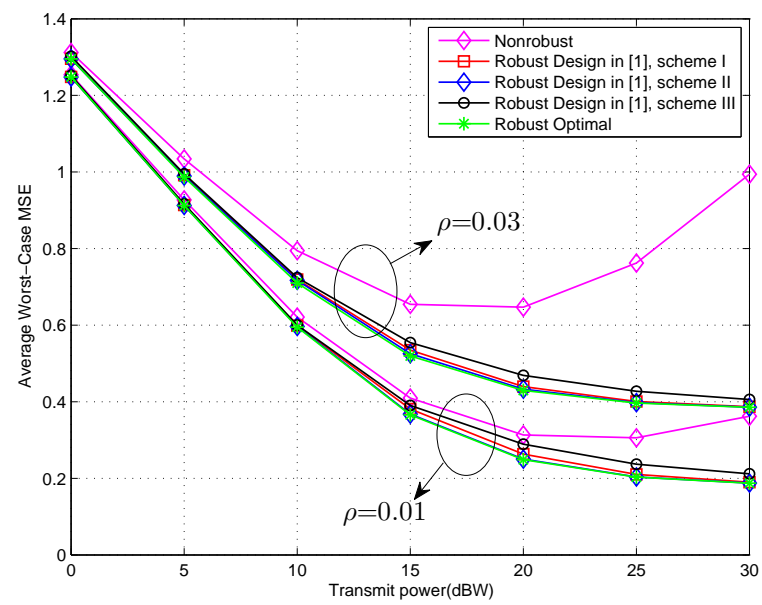

Fig. 3. Worst-case MSE versus different transmit power for $M=N=$ $L=2$.

As an other example, Fig. 3 depicts the average worstcase MSE performance versus different transmit power with $\rho=0.01$ and $\rho=0.03$. We consider the case when $M=N=L=2$. Fig. 3 verifies the superior performance of robust schemes over the non-robust scheme in [5]. Moreover, it can be observed that scheme II has an almost optimal performance, while scheme I approximately approaches to the optimal solution. Therefore, when the advanced software package (such as CVX) is not available, scheme I (or scheme II) can be viewed as a simple implementation of the global optimal method.

\section{CONClusions AND Future Work}

In this paper, we investigate the global optimal transceiver design in the MIMO link under deterministic CSI uncertainty model. We first prove that the optimal design of the transmitter-equalizer has a favorable channel-diagonalizing structure. Then we simplify the original problem into a scalar optimization problem, and obtain the global optimal solution via an SDP problem. Simulation results show that our method outperforms the existing schemes.

We only considered the point-to-point MIMO system in this work. However, as pointed out in [4], the transmission can also be affected by the multiple access interference (MAI), if the transmit-receive nodes are active over a communication network which employs non-orthogonal multiplexing. In this case, the received signal at the destination is contaminated by the spatially colored Gaussian noise and the robust optimal transceiver design must be reconsidered. Hence, it would be interesting to address this issue in our future research.

\section{REFERENCES}

[1] J. Wang and M. Bengtsson, "Joint optimization of the worst-case robust MMSE MIMO transceiver", IEEE Signal Process. Letters, vol.18, no. 5, pp. 295-298, May, 2011.

[2] Z. Wang, and Wen Chen, "Relay beamforming Design with SIC detection for MIMO multi-relay networks with Imperfect CSI," IEEE Trans. Vehicular Technol., vol. 62, no. 8, pp. 3774-3785, Oct. 2013. 
[3] E. Baccarelli, M. Biagi, C. Pelizzoni, and N. Cordeschi, "Optimal MIMO UWB-IR transceiver for Nakagami-fading and Poisson-arrivals", Journal of Communications, vol. 3, no. 1, pp. 27-40, Jan. 2008.

[4] E. Baccarelli, M. Biagi, C. Pelizzoni, N. Cordeschi, "Optimized power allocation for multiantenna systems impaired by multiple access interference and imperfect channel estimation", IEEE Trans. Vehicular Technol., vol. 56, no. 5, pp. 3089-3105, Sep. 2007.

[5] H. Sampath, P. Stoica, and A. Paulraj, "Generalized linear precoder and decoder design for MIMO channels using the weighted MMSE criterion”, IEEE Trans. Commun., vol. 49, no. 12, pp. 2198-2206, Dec. 2001.

[6] D. P. Palomar, J. M. Cioffi, and M. A. Lagunas, "Joint Tx-Rx beamforming design for multicarrier MIMO channels: A unified framework for convex optimization," IEEE Trans. Signal process., vol. 51, no. 9 , pp. 2381-2401, Sep. 2003.

[7] M. Ding and S. D. Blostein, "MIMO minimum total MSE transceiver design with imperfect CSI at both ends", IEEE Trans. Signal Process., vol. 57, no. 3, pp. 1141-1150, Mar. 2009.

[8] X. Zhang, D. P. Palomar, and B. Ottersten, "Statistically robust design of linear MIMO transceivers", IEEE Trans. Signal Process., vol. 56, no. 8, pp. 3678-3689, Aug. 2008.

[9] J. Wang and D. P. Palomar, "Robust MMSE precoding in MIMO channles with pre-fixed receivers", IEEE Trans. Signal Process., vol. 58 , no. 11 , pp. 5802-5818, Nov. 2010.

[10] N. Khaled, S. Thoen, and L. Deneire, "Optimizing the joint transmit and receive MMSE design using mode selection", IEEE Trans. Commun., vol. 53, no. 4, pp. 730-737, Apr. 2005.

[11] H. Shen, J. Wang, B. C. Levy, and C. Zhao, "Robust optimization for amplify-and-forward MIMO relaying from a worst-case perspective," IEEE Transactions Signal Process., vol. 61, no. 21 pp. 5458-5471, Nov., 2013.

[12] H. Shen, J. Wang, W. Xu, Y. Rong, and C. Zhao, "A worst-case robust MMSE transceiver design for nonregenerative MIMO relaying", IEEE Trans. Wireless Commun., vol. 13, no. 2, pp. 695-709, Feb. 2014

[13] H. Tang, W. Chen, J. Li, and H. Wan, "Achieving global optimality for joint source and relay beamforming design in two-hop relay channels," IEEE Trans. Vehicular Technol., vol. 63, no. 9, pp. 4422-4435, Nov., 2014.

[14] S. Boyd, and L. Vandenberghe, "Convex Optimization," Cambridge University Press, 2004.

[15] M. Grant and S. Boyd, CVX' Users' Guide, 2009, [Online], http://cvxr.com/cvx/doc/index.html

[16] L. Vandenberghe and S. Boyd, "Semidefinte programming," SIAM Rev., pp. 49-95, 1996. 